\title{
Comparative Analysis of Gene Expression for Convergent Evolution of Camera Eye Between Octopus and Human
}

\author{
Atsushi Ogura, Kazuho Ikeo, and Takashi Gojobori ${ }^{1}$ \\ Center for Information Biology and DNA Data Bank of Japan, National Institute of Genetics, Mishima, 411-8540, Japan
}

\begin{abstract}
Although the camera eye of the octopus is very similar to that of humans, phylogenetic and embryological analyses have suggested that their camera eyes have been acquired independently. It has been known as a typical example of convergent evolution. To study the molecular basis of convergent evolution of camera eyes, we conducted a comparative analysis of gene expression in octopus and human camera eyes. We sequenced 16,432 ESTs of the octopus eye, leading to 1052 nonredundant genes that have matches in the protein database. Comparing these 1052 genes with 13,303 already-known ESTs of the human eye, 729 (69.3\%) genes were commonly expressed between the human and octopus eyes. On the contrary, when we compared octopus eye ESTs with human connective tissue ESTs, the expression similarity was quite low. To trace the evolutionary changes that are potentially responsible for camera eye formation, we also compared octopus-eye ESTs with the completed genome sequences of other organisms. We found that 1019 out of the 1052 genes had already existed at the common ancestor of bilateria, and 875 genes were conserved between humans and octopuses. It suggests that a larger number of conserved genes and their similar gene expression may be responsible for the convergent evolution of the camera eye.
\end{abstract}

[Supplemental material is available online at www.genome.org.]

The evolution of the eye is one of the most complicated and interesting stories for molecular biologists and molecular evolutionists. Much is known about the photoreceptive organs of various animals (Salvini-Plawn and Mayr 1977; Osorio and Bacon 1994). These organs are highly diverse in structure, ranging from small groups of light-sensitive cells to highly sophisticated and complex structures that register precise images in some groups of arthropods, molluscs, and vertebrates. The single-lens camera eye is found in vertebrates and in two groups of molluscs, octopus and squid. Other molluscs have various types of eye, such as the concave mirror eye and the pinhole eye. Insects, members of Lophotrochozoa, have compound eyes. Despite the differences in direction of visual cells, focusing mechanism, ability to detect polarized light and encoding genes for crystallins, the camera eyes of human and octopus are believed to have independently evolved after the divergence of the two lineages during the Precambrian period because both humans and octopuses have structural similarities in their camera eyes, as shown in Figure 1 (Harris 1997). Therefore, the eyes of humans and octopuses have been described as a typical example of convergent evolution (Fig. 2; Brusca and Brusca 1990; Futuyma 1997). Convergent evolution is the process by which independently evolved features that are superficially similar to each other can arise through different developmental pathways (Lauder 1981).

This view has been changed, however, by Gehring and Ikeo (1999), who maintain that the expression of the common master regulator Pax6 in both types of eyes indicates the divergence of these two types of eyes from a single prototype eye present in the common ancestor of cephalopods and vertebrates. It has previously been reported that Pax6, a "master control" gene for the development of the eye, is highly conserved across species. Within molluscs, it has been shown that the scallop, ear shell,

\footnotetext{
1Corresponding author.

E-MAIL tgojobor@genes.nig.ac.jp; FAX 81-559-81-6848.

Article and publication are at http://www.genome.org/cgi/doi/10.1101/ gr.2268104.
}

and squid all express Pax6 (Tomarev et al. 1997). Pax6 expression has not yet been observed in the octopus. However, the expression of Pax6 in the camera eye of the squid, a member of the same phylum, supports the prediction that Pax6 controls the development of the octopus eye. However, there is no clear explanation of how the elaborate camera eyes of humans and octopuses evolved from the prototype eye. In other words, there is a gap between the evolution of genes expressed in the camera eye and the evolution of morphological structures of the camera eye.

Studying gene expression can lead us to speculate on the biological and functional mechanisms. It is of particular interest to examine the convergent evolution of human and octopus camera eyes from the viewpoint of gene expression, in order to understand the diversification of these organs. Here, we address the question of what kind of gene expressions are maintained in their camera eyes during evolution. Thus, we focus on the gene expression profiles to explain the evolutionary process of camera eyes. For this purpose, we use two approaches. First, we conduct a comparative analysis of gene expression between human and octopus eyes to examine the similarity of gene expression. Second, we estimate the ancestral gene set of the camera eye to determine the number of genes that have been conserved since the divergence of these two lineages from the common ancestor. We then discuss the evolutionary mechanism of convergent evolution of the camera eye from the viewpoint of gene expression profiles.

\section{RESULTS}

\section{Gene Expression Profile of the Octopus Eye}

We made a gene-expression profile based on the occurrence frequency of the mRNAs in the octopus eye. The expressed sequence tag (EST) assembly system was used to obtain 2824 nonredundant sequences from a total of 16,432 clones. To investigate the possible functions of these octopus-eye ESTs, we conducted a 


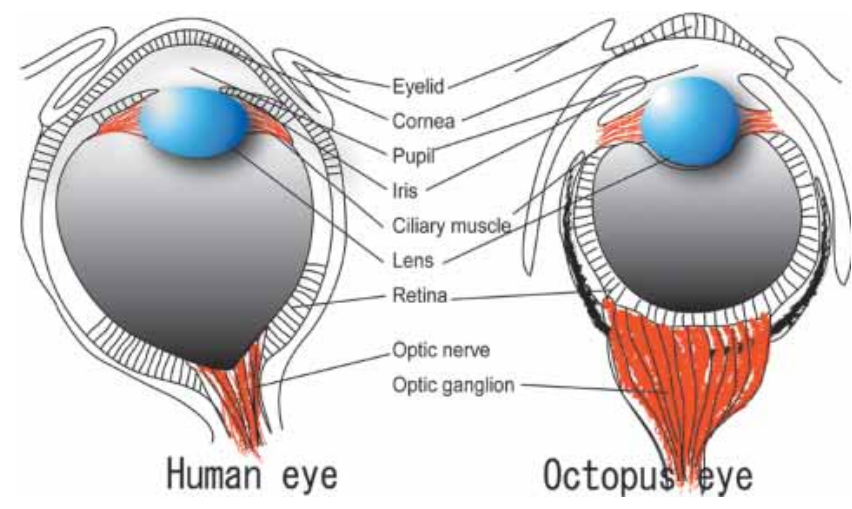

Figure 1 Structural similarities between human and octopus eyes. Even though there are some differences between human and octopus eyes, each of the tissues such as eyelid, cornea, pupil, iris, ciliary muscle, lens, retina, and optic nerve/ganglion corresponds well to each other. The octopus eye forms from an epidermal placode through a series of successive infoldings, whereas the human eye forms from the neural plate and induces the overlying epidermis to form the lens (Harris 1997). The differences in developmental processes between human and octopus are explained in the same reference (Harris 1997). This figure was modified with permission from Sinauer Associates, Inc., (c) 1990 (Brusca and Brusca 1990).

similarity search for identifying homologous sequences in the nonredundant protein database. Using the 2824 sequences as a query, we identified homologs for 1052 of the ESTs (Supplemental Table). This set of 1052 genes was used for the analyses described below, to determine the relationships of the genes expressed in the octopus eye to those that are involved in the developmental process and biological function of the eyes of other species (Table 1). The sequences of octopus-eye ESTs are available online at http://www.cib.nig.ac.jp/dda/database/octopus.htm.

\section{Commonly Expressed Genes in Human and Octopus Camera Eyes}

Using the 1052 nonredundant gene sequences, a comparative analysis of gene expression in human and octopus camera eyes was performed to estimate the number of genes that are commonly expressed in these two lineages. A similarity search was conducted using the set of genes expressed in the octopus eye and the gene-expression data for the human eye obtained from the three databases of BodyMap, the Mammalian Gene Collection (MGC), and the National Eye Institute NEIBank. First, we created a single set of genes from the three distinct databases of gene expression for the human eye (see Methods section). Next, we obtained full-length gene sequences by comparing the geneexpression database with the human gene data from Ensembl (at the European Bioinformatics Institute) and UniGene (at the National Center for Biotechnology Information). We confirmed that 13,303 human-eye ESTs have matches in protein databases. Using the 1052 nonredundant sequences from the octopus eye as query sequences, we performed a BLAST search against the 13,303 human genes. As a result, we identified a total of 729 genes that were commonly expressed in both human and octopus eyes (Fig. 3). It follows that $69.3 \%$ of the 1052 nonredundant sequences from the octopus eye were commonly expressed in the human eye. To test whether these genes are significantly different when the same sequences from the octopus eye are compared with the genes expressed in human tissues other than eyes, we also performed a homology search between octopus-eye ESTs and human-connective-tissue ESTs. To be fair, we used 2430 humanconnective-tissue ESTs as well as 3809 human-eye ESTs from the same database, BodyMap. We then found that the number of genes commonly expressed between the octopus eye and the human connective tissues was only 44 , whereas that of genes between the octopus and human eyes was 162 (Fig. 3). Thus, the former was about four times less than the latter.

Moreover, to investigate the functional characteristics of the commonly expressed genes in human and octopus eyes, we conducted functional annotation of each gene (Table 2). A comprehensive survey of the literature showed that some of the genes have been previously reported to have functions that are related mainly to the eye. Besides, the homologous genes to $\operatorname{six} 3, \ln \times 2$, retinal arrestin, retinal dehydrogenase, $\beta$-catenin, neuronspecific enolase, and human nuclear-transport receptor karyopherin/importin- $\beta$ were found to be expressed in the octopus eye. These genes are known to be important for the formation and function of the vertebrate camera eye. For example, Six 3 is necessary for the patterning of anterior neuroectoderm including the retina (Carl et al. 2002), and $L h \times 2$, which is regulated by Pax6, is also necessary for normal development of the eye, particularly the retina (Porter et al. 1997).

\section{Ancestral Gene Set of the Camera Eye}

To discuss the molecular mechanism of convergent evolution, it is crucial to determine how many expressed genes in the camera eye of the octopus were present in the genome of the last shared ancestor of the two lineages, namely, the common ancestor of bilateria. The ancestral gene set can be estimated by comparing the genes that are expressed in the octopus eye with those that are present in the genomes of deuterostomes and out-group species of bilaterians. If octopus genes have homologs in the deuterostomes or out-group bilaterians, we can reasonably consider that such genes should have existed in the last common ancestor of protostomes and deuterostomes. To estimate the ancestral gene set for the camera eye, we conducted a similarity search by comparing the 1052 nonredundant sequences of octopus-eye ESTs with a total of 1,039,847 genes from the genomes of deuterostomes and out-group bilaterian species. As a result, we identified 1019 out of the 1052 sequences that can be considered as the putative ancestral gene set for the camera eye at the time of the divergence of human and octopus lineages (Fig. 4; Supplemental Table).

\section{Conservation of Camera Eye-Related Genes in Extant Animals}

Because the genomes of modern bilaterians have diverged from their ancestral genome, the molecular changes that led to the evolution of the camera eye in humans and octopuses might be

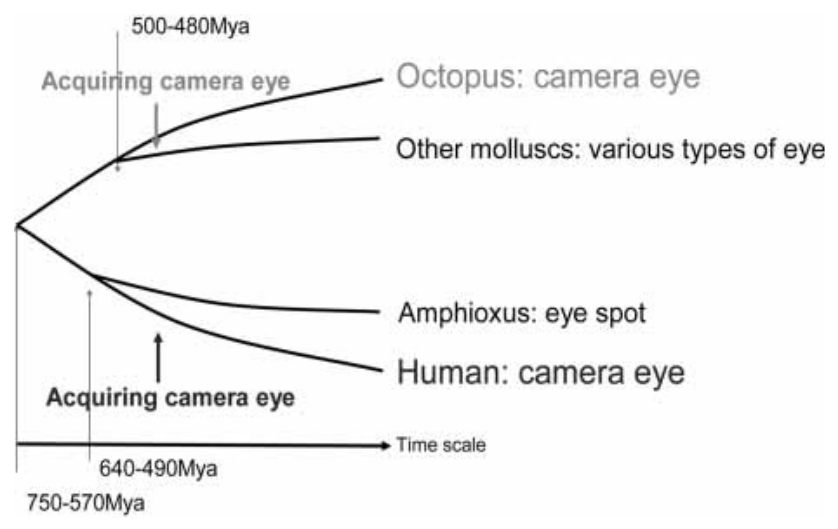

Figure 2 The phylogenetic evidence of convergent evolution of camera eyes between humans and octopuses. Each camera eye has different evolutionary origins.

\section{Genome Research www.genome.org}


Table 1. The Number of Octopus ESTs Obtained in This Study

\begin{tabular}{|c|c|c|}
\hline \multirow[b]{2}{*}{ Categories } & \multicolumn{2}{|c|}{ No. of ESTs } \\
\hline & In whole eye ${ }^{a}$ & In all libraries ${ }^{b}$ \\
\hline Total clones ${ }^{c}$ & 9489 & 16,432 \\
\hline Usable clones $^{d}$ & 5273 & 9448 \\
\hline Nonredundant sequences $\mathrm{e}$ & 1509 & 2824 \\
\hline Not matched to any protein & 898 & 1772 \\
\hline Matched to protein & 611 & $1052^{i}$ \\
\hline Function known ${ }^{f}$ & $454^{\mathrm{h}}$ & 691 \\
\hline Function unknown ${ }^{g}$ & 157 & 361 \\
\hline
\end{tabular}

A summary of CDNA libraries and EST assembly of Octopus eye. ${ }^{a}$ The number of ESTs sequenced from cDNA library A (whole eye).

${ }^{b}$ The number of ESTs sequenced from both CDNA library A (whole eye) and CDNA library B (eyeball without lens).

'Total number of clones that have been sequenced and reported in this paper.

${ }^{\mathrm{d}}$ The number of clones obtained after vector-sequence elimination and quality check.

eThe number of nonredundant sequences.

'The function "known" represents those sequences that match to some proteins in the nonredundant protein database.

9The function "unknown" represents those sequences that do not match to any protein in the nonredundant protein database.

${ }^{h}$ Indicates the genes that were used in the analysis of the functional categorization.

indicates the genes that were used in all analyses in this study except for the functional categorization.

discernable in their genomes. It is possible to speculate on the evolutionary processes that led to the development of the camera eye by estimating the genetic changes that occurred in the ancestral gene set. The availability of partial or complete genome sequences for several species has made it possible to estimate whether or not a particular gene has been conserved during its evolution. In this study, we use the term "evolutionary conservation" in the meaning that the genes had existed in the common ancestor of the organisms compared. Thus, we estimated the number of genes in the putative ancestral gene set for the camera eye that have been evolutionarily conserved among the nematode, fly, and human lineages. First, using the 1019 genes in our proposed ancestral gene set for the camera eye, we conducted a similarity search to infer homology against all the genes in the complete genomes of several species. Then, we estimated the number of genes in the ancestral gene set that had been evolutionarily conserved in the genome. Among the protostomes, we found that 714, 769, and 777 of the 1019 genes in the ancestral gene set were evolutionarily conserved in the genomes of nematodes, mosquitos, and flies, respectively (Fig. 4). In the case of deuterostomes, 834 of the 1019 genes were evolutionarily conserved in the tunicate genome, and 867,865 , and 879 genes were evolutionarily conserved in the vertebrate genomes of Fugu, mouse, and human (Fig. 4). Therefore, it is clear that genes from the proposed ancestral gene set for the camera eye have been evolutionarily conserved for the vertebrate genome in a higher proportion than for the insect or nematode genomes.

\section{The Loss of Genes Related to the Camera Eye}

As mentioned earlier, vertebrates have camera eyes, whereas insects have compound eyes, although nematodes do not have eye structures. Investigating the differences in the ancestral genes that have been conserved in these species, by use of the octopusderived ancestral gene set, we determined whether individual genes might be related to the developmental process and biological function of the camera eye. We carried out a similarity search to infer homology between the ancestral gene set for the camera eye and genes in the genomes of nematode, mosquito, fly, tunicate, Fugu, mouse, and human. The results showed that 646 genes were conserved commonly among all of the species groups examined, indicating that these genes might have important functions. Furthermore, 57, 20, 19, and 7 genes in the ancestral gene set were conserved exclusively in lineages toward vertebrates, tunicates, insects, and worms, respectively (Table 3).

We further investigated the loss of genes from the putative ancestral gene set for the camera eye through the following analysis. Using the patterns of gene conservation described in the previous section, each of the gene-loss events was assigned to a particular branch of the phylogenetic tree. For example, genes that are conserved between humans and flies but not between humans and nematodes were considered to have been lost in nematodes after the divergence of nematode and human lineages. Applying this principle to all of the genes in the proposed ancestral gene set for the camera eye, we assigned all of the potential gene-loss events to the phylogeny. The results showed that 305,250 , and 242 of the 1019 genes in the ancestral gene set $(29.9 \%, 24.5 \%$, and $23.7 \%)$ were lost in nematodes, mosquitoes, and flies, respectively. In contrast, only 142,144 , and 130 of the 1019 genes (12.8\%-14.1\%) were lost in vertebrates ( $F u g u$, mouse, and human; Fig. 4; Table 3).

\section{The Function of Camera Eye-Specific Genes}

We found that 14 out of 57 genes were found only in octopus and all the vertebrates examined, suggesting that these genes might have played indispensable roles of camera eyes (Table 3; Supplemental Table). Moreover, we found that five out of the 14 genes were certainly involved in the function of the camera eyes of humans and octopuses. For example, diacylglycerol kinase $\gamma$, which is expressed in the rod outer segment of the retina, was found in humans and octopuses, but not in the invertebrates examined except octopus (Stohr et al. 1999). For another example, dihydroxyacetonephosphate acyltransferase is the peroxisomal key enzyme of plasmalogen biosynthesis in eye-lens epithelial cells (Thai et al. 1999). This enzyme is also not found in the invertebrates examined except octopus. Moreover, the gelsolin gene is commonly expressed in the whole octopus eye and the cornea of humans

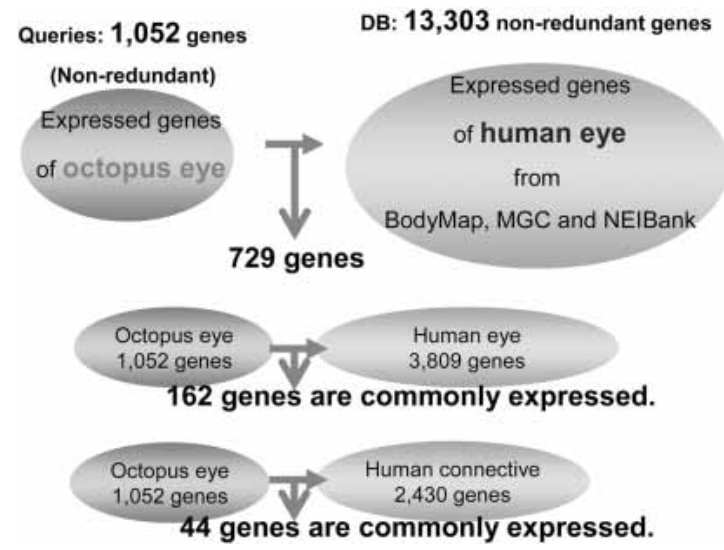

Figure 3 Commonly expressed genes between octopuses and humans. (A) Comparing 1052 nonredundant genes expressed in octopus-eye ESTs with the 13,303 gene set (data from NElbank, MGC, BodyMap) expressed in human eyes, 729 genes were detected as the commonly expressed genes between octopus and human eyes. (B) In all, 162 genes are commonly expressed between octopus and human eyes (data from only BodyMap), whereas only 44 genes are commonly expressed between octopus eyes and human connective tissue (data from BodyMap). 
Table 2. Functional Categorization Using 454 Nonredundant Sequences

\begin{tabular}{lcc}
\hline Molecular functions & $\begin{array}{c}\text { Frequency } \\
(\%)\end{array}$ & $\begin{array}{c}\text { Nonredundant } \\
(\%)\end{array}$ \\
\hline Structural protein & $4324(82.0 \%)$ & $115(25.3 \%)$ \\
Enzyme & $231(4.4 \%)$ & $111(24.4 \%)$ \\
Ligand binding or carrier & $357(6.8 \%)$ & $47(10.4 \%)$ \\
Nucleic acid binding & $96(1.8 \%)$ & $44(9.7 \%)$ \\
Ribosomal protein & $71(1.3 \%)$ & $39(8.6 \%)$ \\
Transporter & $43(0.8 \%)$ & $25(5.5 \%)$ \\
Signal transducer & $60(1.0 \%)$ & $22(4.8 \%)$ \\
Enzyme regulator & $22(0.4 \%)$ & $13(2.9 \%)$ \\
Obsolete & $27(0.5 \%)$ & $10(2.2 \%)$ \\
Chaperone & $12(0.2 \%)$ & $7(1.5 \%)$ \\
Defence/Immunity protein & $10(0.2 \%)$ & $7(1.5 \%)$ \\
Cell adhesion molecule & $6(0.1 \%)$ & $5(1.1 \%)$ \\
Cell cycle regulator & $5(0.1 \%)$ & $4(0.9 \%)$ \\
Apotosis regulator & $6(0.1 \%)$ & $2(0.4 \%)$ \\
Motor & $2(0.0 \%)$ & $2(0.4 \%)$ \\
Chaperone regulator & $1(0.0 \%)$ & $1(0.2 \%)$ \\
Total & $5273(100.0 \%)$ & $454(100.0 \%)$ \\
\hline
\end{tabular}

The most highly expressed genes are those encoding for structural proteins, which are essential for the maintenance of cell and organ structures. In the present study, we used 1509 nonredundant sequences that consisted of 5273 clones obtained from "cDNA library A (whole eye)." This library represents the gene expression of whole octopus eyes. Of the 1509 nonredundant sequences, we assigned the molecular function of gene ontology terms to 454 genes, representing $30 \%$ of 1509 genes. The expression frequency for structural proteins is 4324 times in ESTs, whereas the rest of the 949 times are related to proteins having other functions. Among the expression frequency of 4324 , crystalline genes occupy a large portion, $\sim 40 \%$ of the entire ESTs.

(Xu et al. 2000). The functional validations for the remaining nine out of the 14 genes remain to be conducted in the future study.

\section{DISCUSSION}

\section{Similarity in Gene Expression of Human and Octopus Eyes}

In spite of the evolutionary divergence between octopuses and humans, $69.3 \%$ of the genes examined (729 of the 1052 genes) were commonly expressed in the camera eyes of human and octopus. Moreover, comparison of octopus-eye ESTs with genes in the human connective tissue indicates that the similarity of gene expression between human and octopus eyes should be remarkable. Note that the increase of gene expression similarities between human and octopus eyes from $15 \%(162 / 1052)$ to $69.3 \%$ $(729 / 1052)$ is caused by the increase of the EST data set of human eye from only 3809 ESTs in the database of BodyMap to 13,303 human-eye ESTs in the combined database of NEIbank, MGC, and BodyMap. This observation suggests that many more similarities of gene expression between human and octopus eyes will be observed when the EST data increase further. Although these 729 genes might contain housekeeping genes because the 44 genes were also found in the ESTs of human connective tissues in BodyMap, we found that many more genes (118 genes) were specific to the camera eye in this case. Therefore, we suggest that these 729 genes contain genes necessary for the developmental process and biological function of the camera eye.

From the viewpoint of key genes in eye development, Pax6 has not been found in the octopus. However, a six 3 homolog was present in the set of octopus-eye ESTs that were identified in this study. The six 3 gene is involved in a downstream part of the developmental pathway of eye formation controlled by Pax6. Therefore, it is likely that the Pax6-pathway for eye formation is conserved in the octopus. These observations imply that the gene expression patterns in both the eyes of humans and octopuses are remarkably similar. In other words, the common ancestor of octopus and human had not only the common master regulator, Pax6, but also the ancestral gene set for the camera eyes.

For several reasons, it is unlikely that all of the genes expressed in the octopus eye were identified in this analysis. It is difficult to detect genes that are expressed at a low level. Moreover, the octopus-eye ESTs were generated randomly from the cDNA libraries, which could have been incomplete as a result of mRNA degradation. Furthermore, mRNAs can only be obtained from cells at a particular developmental stage and time. Therefore, it is obvious that we cannot describe all of the genes related to the developmental process or biological function of the camera eye using a sample from an adult octopus. However, even though the genes used in the present study are only a subset of all the expressed genes, it is quite possible that the genes having important roles in the developmental process and biological function are identified through comparative studies of octopus eye ESTs.

\section{Had the Origin of the Gene Set for the Camera Eye Been Formed in the Common Ancestor of Bilateria?}

For the evolutionary origin of the gene set working for the camera eye, we observed that 1019 genes existed in the genome of the common ancestor of the bilaterian animals. Although the morphology of the ancestral eye cannot be inferred from this study, we were able to provide strong support for the hypothesis that these genes having had an important role in the function of camera eyes in both humans and octopuses were present in the last common ancestor of these two lineages. Taking this observation into account, we can reasonably contend that the convergent evolution of camera eyes is caused by the already-abundant

\section{The number of ancestral gene set of camera eye ${ }^{*}: 1,019$}

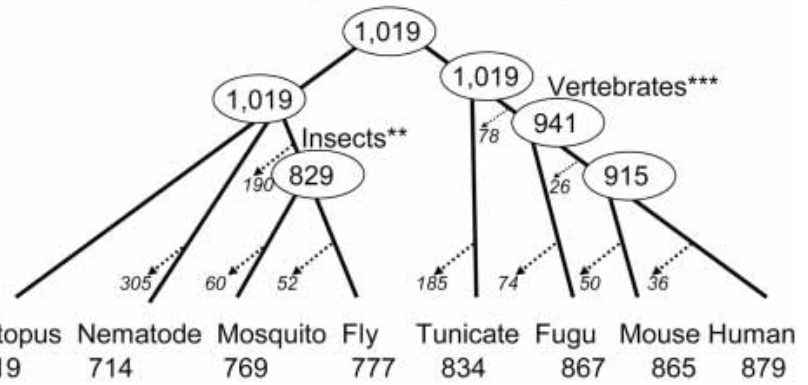

Figure 4 A scheme to illustrate the number of genes derived from the ancestral gene set of the camera eye in each species lineage. This topology has been accepted by many molecular biologists and developmental biologists (Schmidt-Rhaesa 1998; Adoutte et al. 2000; Girbet et al. 2000; Morris 2000; Peterson et al. 2000, 2001). The number of conserved genes is shown below each species name. The numbers in italic represent the number of gene loss in each branch. (*) The number of the ancestral gene set that was obtained from the estimation of homologous genes between octopus-eye ESTs and the genomes of deuterostomes and outgroup bilaterian species. (**) The number of conserved genes shared among the ancestral gene set and the genomes of the mosquito and fly as the representatives of conserved genes in insects. (***) The union of conserved genes in Fugu, mice, and humans as representatives of conserved genes in vertebrates.

\section{Genome Research}


Table 3. Categorization of Conserved Genes From the Viewpoint of Octopus Eye Genes

\begin{tabular}{|c|c|c|c|c|c|}
\hline Categories & Vertebrate & Ciona & Insects & Worm & Tota \\
\hline Common genes $^{\mathrm{a}}$ & 646 & 646 & 646 & 646 & 646 \\
\hline Vertebrate-specific genes ${ }^{b}$ & 57 & - & - & - & 57 \\
\hline Ciona-specific genes $^{\mathrm{b}}$ & - & 20 & - & - & 20 \\
\hline Deutoromes-specific genes ${ }^{b}$ & 35 & 35 & - & - & 35 \\
\hline Lost genes only in vertebrate ${ }^{c}$ & - & 0 & 0 & 0 & 0 \\
\hline Lost genes only in Cionac & 38 & - & 38 & 38 & 38 \\
\hline Lost genes only in insects ${ }^{c}$ & 19 & 19 & - & 19 & 19 \\
\hline Lost genes only in worm ${ }^{c}$ & 104 & 104 & 104 & - & 104 \\
\hline Lost genes in Ciona and worm ${ }^{c}$ & 35 & - & 35 & - & 35 \\
\hline Lost genes in vertebrate and insects ${ }^{c}$ & - & 4 & - & 4 & 4 \\
\hline Lost genes in Ciona and insects ${ }^{c}$ & 7 & - & - & 7 & 7 \\
\hline Lost genes in vertebrate and worm ${ }^{c}$ & - & 6 & 6 & - & 6 \\
\hline Conserved genes in out-group of bilateria ${ }^{d}$ & - & - & - & - & 48 \\
\hline Conserved genes from ancestral gene set & 941 & 834 & 829 & 714 & 1019 \\
\hline Shared genes between octopus and insects ${ }^{\mathrm{e}}$ & - & - & 19 & - & 19 \\
\hline Shared genes between octopus and worm ${ }^{e}$ & - & - & - & 7 & 7 \\
\hline Shared genes in Protostomes lineage ${ }^{\mathrm{e}}$ & - & - & 7 & 7 & 7 \\
\hline Shared genes between octopus and other species & 94 & 834 & 855 & 728 & 1052 \\
\hline
\end{tabular}

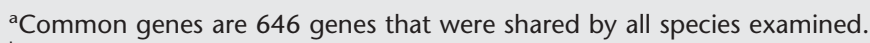

bSpecific genes: some of these are genes conserved only in specific lineages, and especially 57 from vertebrate-specific genes, may have been related to the camera eye.

'Lost genes are genes that have been lost in the specific lineages.

${ }^{\mathrm{d} C o n s e r v e d ~ g e n e s ~ i n ~ o u t-g r o u p ~ o f ~ b i l a t e r i a ~ a r e ~ g e n e s ~ m a t c h e d ~ t o ~ p r o t e i n s ~ b e l o n g i n g ~ t o ~ t h e ~ o u t-g r o u p ~ o f ~ b i l a t e r i a n ~ s p e c i e s ~ b u t ~ t h a t ~ d o ~ n o t ~ m a t c h ~}$ to proteins belonging to bilaterian species.

eThese categories are not included in the ancestral gene set because they seem to have emerged after the divergence of protostomes and deuterostomes.

presence of the commonly shared genes as the ancestral gene set and the remarkable similarity of expression profiles of their derived genes.

Moreover, the numbers of conserved genes in insects and nematodes were less than those in vertebrates; nevertheless, insects and nematodes are more closely related to octopuses than are vertebrates. This indicates that insects and nematodes have lost many more genes in their lineages than humans have. On the other hand, the number of conserved genes in tunicates is larger than those in insects or worms, even though they also do not possess the camera eye structure. One of the possible reasons is that insects or nematodes have lost the genes possibly unimportant for their body plans, because these organisms are known to tend to lose the genes unless they are important (Fig. 3). As we estimated 1019 genes for ancestral genes for the camera eye, more than 760 genes were conserved in flies or worms. This conservation in flies or worms suggests that not all of these genes were specific to the camera eye. In the case of conserved genes in flies or worms, it is possible that these genes derived from the common ancestral gene set can be used in other organs and cells such as the photo-sensory system.

To test whether ancestral genes for the camera eye include duplicated genes after octopus speciation and to evaluate the influence of such duplicated genes, if any, on the evolutionary process, we first estimated the number of lineage-specific duplications in octopus lineages. Then, we subtracted the number of the possible duplicated genes from the total number of the ancestral genes for the camera eye. As a result, we observed that the number of ancestral genes, the conserved genes in humans, the conserved genes in flies, and the conserved genes in worms decreased from 1019, 879, 777, and 714 to $943,806,708$, and 660, respectively, indicating only a small number of decreased genes. Therefore, the duplicated genes might not affect our discussion much.

In this study, we assumed the phylogeny in which flies are closer to worms than vertebrates. However, there is an alternative hypothesis in which flies are closer to vertebrates rather than worms (Blair et al. 2002). To test the influence of this alternative phylogeny on our study, we have computed the number of the ancestral gene set for the camera eye and the number of evolutionarily conserved genes in the extant animals under the assumption of the alternative phylogeny. As a result, 951 genes were estimated as the ancestral gene set for the camera eye, and 816,685 , and 768 genes were conserved in humans, flies, and Ciona, respectively. Therefore, the alternative phylogeny also did not affect our discussion much.

Furthermore, it is also possible that a bias in the total number of genes in the database used in the present study would affect the estimated number of the ancestral gene set for the camera eye, because humans and mice have many more genes in the database than flies or worms. However, this possibility can be reasonably ruled out because we used all the genes deduced in the complete genome for the species examined in the present study.

\section{The Evolutionary Role of Gene Loss}

There are some reports of gene-loss events in vertebrates (Ruddle et al. 1994; Nadeau and Sankoff 1997). However, the relationship between gene-loss events and evolutionary formation of any organ or tissue has been unclear. We found that some of the genes conserved between humans and octopuses have been lost in the organisms having no camera eye structures. This result supports a hypothesis that the genes required for the development and maintenance of the camera eye were retained in the vertebrate and octopus lineages, but lost in insects, nematodes, and tunicates. For insects, it is likely that the genes having functions specific to the camera eye were not important for the evolution of the compound eye, and therefore must have been lost from the genome. For nematodes, eye- 
specific genes might also have been lost. Estimation of gene-loss events from the ancestral gene set allows us to identify genes that were necessary for the evolution and maintenance of the camera eye (Fig. 4). Thus, the patterns of gene loss discussed here might have had an important role in the diversification of the eye. In the future, these genes are required to have experimental validation to elucidate the functional and evolutionary roles for the camera eyes.

\section{Molecular Mechanism of Convergent Evolution of the Camera Eye}

In this study, we used the eye of an adult octopus instead of octopus eyes at the different developmental stages. This is because our prime question is to know what kind of gene expression profiles are maintained in the camera eye between these distantly related species regardless of the developmental stages. Even if the master control gene might have dominated the evolution of the eye, it is the first basis to understand all the genes that are expressed in the camera eyes of an adult form, avoiding any complications caused by the developmental stages. Thus, it is important to discuss how distantly related species evolved these similar organs from the viewpoint of the gene expression profile of adult eyes. In this context, we have shown that 941 genes are shared between vertebrates and octopuses. In fact, 879 genes are shared between humans and octopuses. In contrast, for nematodes and insects that are phylogenetically closer to octopuses, a smaller number of genes (728 and 802 genes, respectively) are shared with the octopus. This implies that the genes involved in the development and function of the camera eye were already present in the shared common ancestor at the time of the divergence of protostomes and deuterostomes. Although we were unable to assign function to 1772 genes of the octopus eye examined in this study, we identified 1052 genes that might have functions in the development and maintenance of the camera eye.

Our results indicate that most of the genes, including several gene pathways necessary for the evolution of the camera eye, might be shared between human and octopus lineages. Therefore, there is strong evidence that the evolutionary mechanisms for the camera eyes of humans and octopuses are subjected to similar gene expression profiles of the commonly conserved gene set, although the developmental processes of the human and octopus eyes are a bit different. We believe that these gene expression similarities could be the bridge of understanding from the genetic system of eye evolution to the developmental process of the camera eyes of humans and octopuses. In future studies, the functional validation of evolutionary events responsible for camera eye formation in both lineages will help us to understand how the elaborate camera eye evolved from the primitive eye that must have had the ancestral gene set for the camera eye.

\section{METHODS}

\section{Animals}

Octopus vulgaris, a Japanese madako captured from the Suruga Bay population, which belongs to the phylum Mollusca and class Cephalopoda, was used for the following studies. It was commercially obtained from the Numazu fish market.

\section{Construction of cDNA Library for the Octopus Eye}

We constructed two types of cDNA libraries of the octopus eye, cDNA library A and B. First, we constructed the cDNA library (A) from the whole part of the octopus eye for studying a gene expression profile in an octopus adult eye. A whole eye is composed of eight different tissues; cliary body, fovea, iris, retina, lens, cornea, supporting muscle, and optic nerve. The cDNA library A (whole eye) has been expected to reflect the frequencies of different mRNAs of adult octopus eyes in vivo. Second, the mRNAs extracted from a whole eye of octopus without a retina and a lens were used for the construction of the cDNA library B. The purpose of constructing the cDNA library B (eyeball without lens) is to increase the nonredundant EST sequences of octopus eye because the cDNA library A (whole eye) contains many redundant clones due to the highly expressed genes such as crystalline genes. We sequenced 5'-ESTs of 9489 clones and 6943 clones from the "cDNA library A (whole eye)" and "cDNA library B (eyeball without lens)," respectively. Finally, we merged the EST data of these two cDNA libraries into a single set of EST data, so that we successfully obtained as many nonredundant sequences as possible for the comparative analysis (Table 1).

\section{Making Nonredundant Sequences and Assembling Sequences}

Nonredundant sequences were constructed through EST assembling for the extending sequences, and gene expression frequencies were calculated in the following protocol. First, EST sequences produced by the ABI 3700 sequencer were translated into a FASTA format from chromatogram files using Phred and Phd2fasta (Ewing et al. 1998). The quality value of each nucleotide was also calculated. Vector sequences were then screened out by cross-match (Ewing et al. 1998), and low-quality sequences were eliminated by Quality Checker, which we developed. As a threshold, the quality value of $<20$ was adopted as the critical threshold, meaning that a reliability of $90 \%$ was guaranteed at the sequence level. In the next step, the gene-clustering method using BLASTCLUST (Altschul et al. 1990) was conducted to obtain a rough estimation of clusters containing similar sequences. ESTs belonging to a certain cluster are expected to share the common ancestor, whether these ESTs could be a part of the same sequence or belong to the same gene family. The minimum length coverage of $<0.3$ and similarity threshold of $<0.3$ were used as criteria for the estimation of gene clusters. For each gene cluster, we assembled sequences using Phrap (Ewing et al. 1998). The number of genes belonging to a particular gene cluster can be considered to be its gene expression frequency. We classified the genes into two categories; (1) a category for contigs belonging to clusters and used for making assembled sequences, and (2) a category for orphans belonging to none of the clusters. The above-mentioned protocol was systematically performed using both Perl and shell scripts.

\section{Homology Search}

BLASTN was used for making nonredundant data sets, assembling the sequences, and clustering the genes. TBLASTX was also used to find homologous sequences among remotely related species such as octopus and human. The threshold value, $e-04$, was used in the following studies because we found this value the best after the reliability test of this threshold value when changing the $e$-value from $e-03$ to $e-100$.

\section{Functional Annotation}

Functional annotation has been conducted as follows: We conducted homology searches using the nonredundant data set of the octopus-eye ESTs that were obtained from all the cDNA libraries constructed. Gene ontology was used for categorizing octopus-eye ESTs. Gene ontology is defined by the Gene Ontology Consortium (Ashburner et al. 2000; The Gene Ontology Consortium 2001). Gene ontology includes three categorizations with respect to gene function: (1) molecular function, (2) biological process, and (3) cellular component. We adopted the molecular function for categorizing genes of the octopus eye. Investigation based on PubMed was conducted for the functional categorization of octopus-eye ESTs. The gene annotation system was con-

\section{Genome Research}


structed as follows: ESTs having homology to known proteins with a probability of $e-04$ and sequence overlap of $70 \%$ were defined as "known proteins." ESTs having homology to unknown proteins with a probability of $e-04$ and sequence overlap of $70 \%$ were defined as "unknown proteins." Other ESTs were considered as no-match ESTs.

\section{Databases for Gene Expression in the Human Eye}

BodyMap is the project for identifying the genes expressed in human cells categorized by tissues (Okubo et al. 1992). The cDNA clones in all the libraries have been sequenced from the $3^{\prime}$ regions. To compare the expressed genes between human and octopus eyes, using the BodyMap data and the human genome sequence data, we obtained $\sim 1800$ genes that were expressed in human eyes (retina, cornea, etc.). MGC, the Mammalian Gene Collection (Strausberg et al. 1999) maintained by NIH, also has a similar data set of genes expressed in several kinds of human tissues. All the data have references to the full-length cDNA database. To compare the expressed genes between human and octopus eyes, using the MGC data, we selected 1200 genes that were expressed in the human eye. NEIbank, maintained by the National Eye Institute, one of the NIH divisions (USA), contains data for several new human-eye cDNA libraries (Graeme 2002). This databank includes a tissue-separated database of Ciliary Body, Cornea, Fovea, Iris, Lens, Optic Nerve, Retina, RPE Choroids, Trabecular Meshwork, and others. In the comparative analyses of gene expression using BodyMap data, we used retina, corneal endothelium, cornea, and iris as human-eye ESTs. We also used fat, aorta, osteoblast, reaming bone, fibroblast, itoh cell, mesangial cell, and muscle as human-connective-tissue ESTs.

\section{ACKNOWLEDGMENTS}

We express our gratitude to Yumi Takezawa, Hiroaki Yamamoto, Chi-Chiu Wang, and Jung-Shan Hwang for their help, suggestions, and discussion. This study was supported, in part, by a grant to T.G. from MEXT (Ministry of Education, Sports, Culture, Science and Technology) of Japan.

The publication costs of this article were defrayed in part by payment of page charges. This article must therefore be hereby marked "advertisement" in accordance with 18 USC section 1734 solely to indicate this fact.

\section{REFERENCES}

Adoutte, A., Balavoine, G., Lartillot, N., Lespinet, O., Prud'homme, B., and De Rosa, R. 2000. The new animal phylogeny: Reliability and implications. Proc. Natl. Acad. Sci. 97: 4453-4456.

Altschul, S.F., Gish, W., Miller, W., Myers, E.W., and Lipman, D.J. 1990. Basic local alignment search tool. J. Mol. Biol. 215: 403-410.

Ashburner, M., Ball, C.A., Blake, J.A., Botstein, D., Butler, H., Cherry, J.M., Davis, A.P., Dolinski, K., Dwight, S.S., Eppig, J.T., et al. 2000. Gene ontology: Tool for the unification of biology. The Gene Ontology Consortium. Nat. Genet. 25: 25-29.

Blair, J.E., Ikeo, K., Gojobori, T., and Hedges, S.B. 2002. The evolutionary position of nematodes. BMC Evol. Biol. 2: 7.

Brusca, R.C. and Brusca, G.J. 1990. Invertebrates. Sinauer Associates, Inc., Sunderland, MA

Carl, M., Loosli, F., and Wittbrodt, J. 2002. Six3 inactivation reveals its essential role for the formation and patterning of the vertebrate eye. Development 129: 4057-4063.

Ewing, B., Hillier, L., Wendl, M., and Green, P. 1998. Basecalling of automated sequencer traces using phred I. II. Error probabilities. Genome Res. 8: 175-185.
Futuyma, D.J. 1997. Evolutionary biology. Sinauer Associates, Inc., Sunderland, MA.

Gehring, W.J. and Ikeo, K. 1999. Pax 6: Mastering eye morphogenesis and eye evolution. Trends Genet. 15: 371-377.

The Gene Ontology Consortium. 2001. Creating the gene ontology resource: Design and implementation. Genome Res. 11: 1425-1433.

Girbet, G., Distel, D.L., Polz, M., Sterrer, W., and Wheeler, W.C. 2000. Triploblastic relationships with emphasis on the acoelomates and the position of Gnathostomulida, Cycliophora, Plathelminthes, and Chaetognatha: A combined approach of $18 \mathrm{~S}$ rDNA sequences and morphology. Syst. Biol. 49: 539-562.

Graeme, W. 2002. A project for ocular bioinformatics: NEIBank Mol. Vis. 8: $161-163$.

Harris, W.A. 1997. Pax-6: Where to be conserved is not conservative. Proc. Natl. Acad. Sci. 94: 2098-2100.

Lauder, G.V. 1981. Form and function: Structural analysis in evolutionary morphology. Paleobiology 7: 430-442.

Morris, S.C. 2000. The Cambrian "explosion" slow-fuse or megatonnage? Proc. Natl. Acad. Sci. 97: 4426-4429.

Nadeau, J.H. and Sankoff, D. 1997. Comparable rates of gene loss and functional divergence after genome duplications early in vertebrate evolution. Genetics 147: 1259-1266.

Okubo, K., Hori, N., Matoba, R., Niiyama, T., Fukushima, A., Kojima, Y., and Matsubara, K. 1992. Large scale cDNA sequencing for analysis of quantitative and qualitative aspects of gene expression. Nat. Genet. 2: 173-179.

Osorio, D. and Bacon, J.P. 1994. A good eye for arthropod evolution. Bioessays 16: 419-424.

Peterson, K.J. and Eernisse, D.J. 2001 Animal phylogeny and the ancestry of bilaterians: Inference from morphology and 18s rDNA sequences. Evol. Dev. 3: 170-205.

Peterson, K.J., Cameron, R.A., and Davidson, E.H. 2000. Bilaterian origins: Significance of new experimental observations. Dev. Biol. 219: 1-17.

Porter, F.D., Drago, J., Xu, Y., Cheema, S.S., Wassif, C., Huang, S.P., Lee, E., Grinberg, A., Massalas, J.S., Bodine, D., et al. 1997. Lhx2, a LIM homeobox gene, is required for eye, forebrain, and definitive erythrocyte development. Development 124: 2935-2944.

Ruddle, F.H., Bentley, K.L., Murtha, M.T., and Risch, N. 1994. Gene loss and gain in the evolution of the vertebrates. Dev. Suppl. 155-161.

Salvini-Plawn, L.V. and Mayr, E. 1977. On the evolution of photoreceptors and eyes. Evol. Biol. 10: 207-263.

Schmidt-Rhaesa, A. 1998. Phylogenetic relationships of the nematomorpha-A discussion of current hypothesis. Zool. Anz. 236: 203-216

Stohr, H., Klein, J., Gehrig, A., Koehler, M.R., Jurklies, B., Kellner, U., Leo-Kottler, B., Schmid, M., and Weber, B.H. 1999. Mapping and genomic characterization of the gene encoding diacylglycerol kinase $\gamma$ (DAGK3): Assessment of its role in dominant optic atrophy (OPA1). Hum. Genet. 104: 99-105.

Strausberg, R.L., Feingold, E.A., Klausner, R.D., and Collins, F.S. 1999. The Mammalian Gene Collection. Science 286: 455-457.

Thai, T.P., Rodemer, C., Worsch, J., Hunziker, A., Gorgas, K., and Just, W.W. 1999. Synthesis of plasmalogens in eye lens epithelial cells. FEBS Lett. 456: 263-268.

Tomarev, S.I., Callaerts, P., Kos, L., Zinovieva, R., Halder, G., Gehring, W., and Piatigorsky, J. 1997. Squid Pax-6 and eye development. Proc. Natl. Acad. Sci. 94: 2421-2426.

Xu, Y.S., Kantorow, M., Davis, J., and Piatigorsky, J. 2000. Evidence for gelsolin as a corneal crystallin in zebrafish. J. Biol. Chem. 275: 24645-24652.

\section{WEB SITE REFERENCES}

http://www.cib.nig.ac.jp/dda/database/octopus.htm; sequences of octopus-eye ESTs.

Received December 14, 2003; accepted in revised form May 12, 2004. 


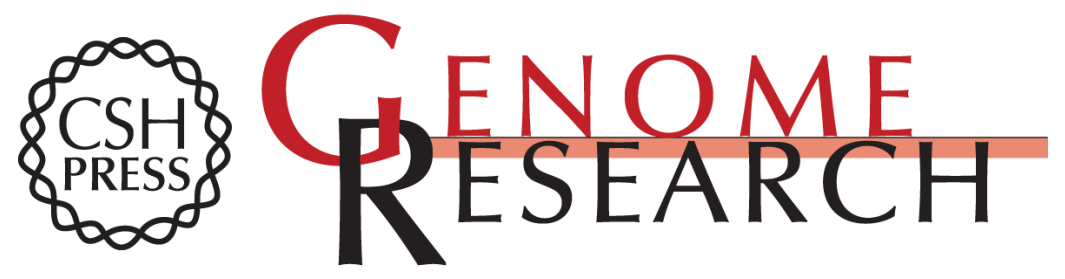

\section{Comparative Analysis of Gene Expression for Convergent Evolution of Camera Eye Between Octopus and Human}

Atsushi Ogura, Kazuho Ikeo and Takashi Gojobori

Genome Res. 2004 14: 1555-1561

Access the most recent version at doi:10.1101/gr.2268104

Supplemental Material

References

License

Email Alerting Service
http://genome.cshlp.org/content/suppl/2004/07/09/14.8.1555.DC1

This article cites 26 articles, 12 of which can be accessed free at: http://genome.cshlp.org/content/14/8/1555.full.html\#ref-list-1

Receive free email alerts when new articles cite this article - sign up in the box at the top right corner of the article or click here.

\section{Affordable, Accurate Sequencing.}

